(PYF), 19 patients experienced a serious adverse event (13.6 per 100 PYF).

Conclusions We have shown in a large real-world cohort of complex, treatment refractory CD patients that UST is a safe and effective treatment option

\section{P136 IMMUNOMODULATORY MECHANISMS OF FMT IS ASSOCIATED WITH CLINICAL RESPONSE IN UC - RESULTS FROM STOP-COLITIS}

\begin{abstract}
${ }^{1}$ Mohammed Nabil Quraishi*, ${ }^{2}{ }_{\text {Ye }} 00,{ }^{3}$ Andrew D Beggs, ${ }^{2}$ David Withers, ${ }^{3}$ Animesh Acharjee, ${ }^{1}$ Naveen Sharma, ${ }^{1}$ Susan Manzoor, ${ }^{4}$ Nicholas J Loman, ${ }^{5}$ Konstantinos Gerasimidis, ${ }^{6}$ Richard Hansen, ${ }^{3}$ Georgios Gkoutous, ${ }^{7}$ Ailsa L Hart, ${ }^{8}$ Daniel R Gaya, 'Tariq H Iqbal. 'University of Birmingham Microbiome Treatment Centre, Birmingham, UK; ${ }^{2}$ Institute of Immunology and Immunotherapy, University of Birmingham, UK, UK; ${ }^{3}$ Institute of Cancer and Genomic Sciences, University of Birmingham, UK; ${ }^{4}$ Institute of Microbiology and Infection, University of Birmingham, UK; ${ }^{5}$ Human Nutrition, University of Glasgow, Glasgow, UK; ${ }^{6}$ Department of Paediatric Gastroenterology, Royal Hospital for Children Glasgow, Glasgow, UK; ${ }^{7} I B D$ Unit, St Marks Hospital, London, UK; ${ }^{8}$ Gastroenterology Unit, Glasgow Royal Infirmary, Glasgow, UK
\end{abstract}

\subsection{6/gutjnl-2020-bsgcampus.211}

Background Studies of faecal microbiota transplantation (FMT) for treating ulcerative (UC) have shown promising results, however mechanisms by which FMT modulates inflammation remain unexplored. Through an open-label pilot of FMT in UC (STOP-Colitis) we conducted a sub-study to explore changes in host colonic mucosal immune cell subsets and gene expression following FMT.

Methods Patients in this study received 8 infusions of FMT over an 8 week period. Colon biopsies were obtained at baseline and at end of the study. Immunophenotyping of colonic lamina propria mononuclear cells (LPMC) and RNA sequencing was conducted on colon biopsies for differential gene expression analysis.

Results 17 patients were recruited to this sub-study of which 12 completed study per protocol. Response (reduction in MAYO score) was seen in $67 \%(8 / 12)$ of patients. Analysis of colonic LPMC populations revealed a significant increase in regulatory $\mathrm{T}$ cells (Tregs, CD4+CD25+CD127lowFoxP3+; $\Delta 5.02 \%$; $\mathrm{p}<0.01)$, effector Tregs (CD4+CD25+CD127CCR7-CD45RA-; $\Delta 12 \% ; \mathrm{p}<0.001$ ), gut homing Tregs (CD4 $+\mathrm{CD} 25+\mathrm{CD} 127-\mathrm{CCR} 7-\mathrm{CD} 45 \mathrm{RA}-\alpha 4+; \quad \Delta 18.55 \% ; \quad \mathrm{p}<0.01)$ and IL-10 producing CD4 cells $(\Delta 2.16 \% ; \mathrm{p}=0.04)$ in responders following FMT. There was a significant reduction in mucosal Th17 (CD4+CD161+CCR6+; $\Delta-7.61 \% ; \mathrm{p}=0.017$ ), IL-17 producing CD4 $(\Delta 7.69 \% ; p=0.05)$ and CD8 $(\Delta-5.18 \%$; $\mathrm{p}=0.04)$ populations in FMT responders. Colonic mucosal gene expression and pathway analysis demonstrated that response to FMT was associated with significant downregulation of host antimicrobial defence response mainly REG and defensin family of anti-microbial peptides, pathogen-associated molecular pattern receptors, MHC class II antigen presentation genes and proinflammatory pathways. There was significant upregulation of butanoate and propionate metabolic pathways in FMT responders.

Conclusion Response to FMT is associated with a significant increase in mucosal gut homing Tregs and butanoate metabolism along with a reduction in Th17 cells and multiple antimicrobial defence and proinflammatory pathways. Exploring microbial mediators in FMT which influence immunometabolism are now under investigation to underpin novel biotherapeutic approaches.

\section{P137 THE IMPACT OF IBD FATIGUE ON HEALTH-RELATED QUALITY OF LIFE: A QUALITATIVE SEMI-STRUCTURED INTERVIEW STUDY}

${ }^{1}$ Shellie Radford*, ${ }^{2}$ Wladyslawa Czuber-Dochan, ${ }^{1}$ Gordon W Moran. ${ }^{1}$ NIHR Nottingham Biomedical Research Centre and University Of Nottingham, Nottingham, UK; ${ }^{2}$ Kings College London, Florence Nightingale Faculty of Nursing, midwifery and palliative care, London, UK

\subsection{6/gutjnl-2020-bsgcampus.212}

Background Fatigue is a frequently reported symptom of inflammatory bowel disease (IBD), experienced by patients with active disease and in remission. Fatigue related to chronic conditions plays a significant negative role in Health-Related Quality of Life (HRQoL), but patients' experience of this have not been described in IBD. We aimed to explore experience of IBD fatigue and its impact on HRQoL in adults diagnosed with IBD.

Methods Qualitative, semi-structured in-depth interviews were conducted with adults with IBD in remission, recruited from out-patient clinics in the United Kingdom. Eligibility and medical history were confirmed at recruitment. Interviews were audio-recorded and transcribed verbatim. Thematic analysis was employed to analyse the data using NVivo 12 software.

Results Fourteen participants (eight females, average age 37.3 years old, range 21-64) were interviewed. All identified themselves as a 'White British', average length of living with IBD fatigue was 10.9 years (range 9 months -17 years). Twelve participants reported that fatigue was constant and two reported intermittent fatigue. There were three key themes that reflect the patient experience: 1) 'The new normal' established through attempts to adapt daily life and acceptance of IBD fatigue impact on daily life and therefore impact on HRQoL. HRQoL is negatively impacted by lack of feelings of fulfilment, not being able to continue on as before the onset of IBD fatigue and a negative perception of self in comparison to others without IBD fatigue; 2) 'Energy as a resource' describes participants attempts to better manage fatigue on a daily basis through planning and prioritising tasks, often prioritising employment or education above social or leisure activities; 3) 'Keeping healthy' encompasses participants beliefs that good nutrition, good general health and keeping active allow them to generate energy more easily allowing some situational control where they have little control over IBD symptoms and subsequently improve HRQoL. Participants reported a mix of physical activities that improved HRQoL, however none reported a specific programme of exercise.

Conclusion Adults with IBD fatigue try to establish a sense of 'new' normality, through maintaining the same or similar, level of activities related to employment or education. However, this is often at the expense of personal, social and leisure activities. The study also indicates that perceptions of conservation of energy through planning and prioritising tasks and high levels of social support were associated with better selfreported HRQoL. Further research is required to explore physical activity-based intervention in relation to IBD fatigue, with use of validated fatigue and HRQoL measures. 